\title{
Modeling Relationships Between Iron Status, Behavior, And Brain Electrophysiology: Evidence From a Randomized Study Involving a Biofortified Grain in Indian Adolescents
}

\author{
Michael J. Wenger ( $\nabla$ michael.j.wenger@ou.edu ) \\ The University of Oklahoma \\ Laura E. Murray Kolb \\ Purdue University \\ Samuel P. Scott \\ International Food Policy Research Institute \\ Erick Boy \\ HarvestPlus, International Food Policy Research Institute \\ Jere D Haas \\ Cornell University
}

\section{Research Article}

Keywords: iron deficiency, memory, cognition, brain, electroencephelography

Posted Date: September 30th, 2021

DOI: https://doi.org/10.21203/rs.3.rs-882215/v1

License: (c) (1) This work is licensed under a Creative Commons Attribution 4.0 International License.

Read Full License 


\title{
Modeling relationships between iron status, behavior, and brain electrophysiology: Evidence from a randomized study involving a biofortified grain in Indian adolescents
}

\author{
Michael J. Wenger ${ }^{1,2^{*}}$, Laura E. Murray Kolb ${ }^{3}$, Samuel P. Scott ${ }^{4}$, Erick Boy ${ }^{5}$ and Jere D. Haas ${ }^{2}$
}

\author{
${ }^{*}$ Correspondence: \\ michael.j.wenger@ou.edu \\ ${ }^{1}$ Department of Psychology, \\ Cellular and Behavioral \\ Neurobiology, The University of \\ Oklahoma, Norman, OK, USA \\ Full list of author information is \\ available at the end of the article
}

\begin{abstract}
Background: Iron deficiency (ID) and iron deficiency anemia (IDA) are highly-prevalent nutrient deficiencies and have been shown to have a range of negative effects on cognition and brain function. Human intervention studies including measures at three levels-blood, brain, and behavior-are rare and our objective was to model the relationships among measures at these three levels in school-going Indian adolescents.
\end{abstract}

Methods: Male and female adolescents in rural India were screened for ID/IDA. Subjects consumed 2 meals/day for 6 months; half were randomly assigned to consume meals made from a standard grain (pearl millet) and half consumed meals made from an iron biofortified pearl millet (BPM). Prior to and then at the conclusion of the feeding trial, they completed a set of cognitive tests with concurrent electroencephalography (EEG).

Results: Consumption of the BPM resulted in significantly larger improvements (relative to the comparison pearl millet) in the iron biomarkers (mean partial $\eta^{2}$ for treatment condition $=0.07$ ), the behavioral variables (mean partial $\eta^{2}=$ 0.18 ), and the EEG variables (mean partial $\eta^{2}=0.21$ ). Critically, the best model for the relationship between iron status and cognition had brain measures as a mediating factor, with both serum ferritin as a primary predictor and hemoglobin as a moderator.

Conclusions: A dietary intervention involving a biofortified staple grain was shown to be effective in improving blood iron biomarkers, behavioral measures of cognition, and EEG measures of brain function. Modeling the relationships among these variables strongly suggests multiple mechanisms by which blood iron level affects brain function and cognition.

Trial registration: Registered at ClinicalTrials.gov: NCT02152150, 02 June 2014.

Keywords: iron deficiency; memory; cognition; brain; electroencephelography

\section{Introduction}

Iron deficiency (ID) and iron deficiency anemia (IDA) are highly-prevalent nutrient deficiencies that can be found at high rates in developing and developed countries [1]. They have been shown to have negative effects on physical performance $[2,3]$ and work productivity $[4,5]$, and there is accumulating evidence that ID, without anemia, has a range of negative effects on cognition and brain function $[6,7,8,9$, $10,11]$, including evidence that ID has a significant negative impact on academic 
performance [12]. However, the mechanisms by which ID exerts its negative impact in humans are not completely clear. In part this is because (a) only a small number of studies have collected both behavioral and neurophysiological data, and (b) an even smaller subset of those studies have attempted to model the hypothesis that changes in brain function mediate the relationships between changes in iron status and changes in behavioral measures of cognition.

Two aspects of brain function seem likely as mechanisms for ID-related cognitive deficits in otherwise healthy children and adults. The first is the effect of variations in iron levels on the neurotransmitter dopamine (DA), in particular the DA D2 receptor and DA transporter (DAT) in animal models [13, 14, 15]. Although there have been no direct measurements of the effects of ID or IDA on DA in humans, there is suggestive indirect evidence, including reductions in spontaneous blink rates in infants with IDA [16], with blink rates being related to levels of central DA [17]. Furthermore, there is evidence from both disease states and pharmacological manipulations that variations in DA levels have distinct effects on controlled attention and memory $[18,19,20]$. The second candidate is oxygen transport, given that the brain's oxygen demands are quite high and that task-dependent changes in cerebral blood flow seem to reflect a generalized mobilization of brain resources [21, 22, 23].[1]

In the present study, our measures of cognition included change in a set of behavioral tasks, including measures of controlled attention and memory, that we have shown to be sensitive to variations in iron status in adolescents and women of reproductive age $[6,8,9]$. Our measures of mediating brain function included a set of electroencephalographic (EEG) features that have been shown to be related to aspects of attention and memory retrieval, including power in the $\alpha$ and $\gamma$ frequency bands [28, 29], and the amplitude of the N1 and P3 components [10, 11]. As our primary measure of iron status, we used serum ferritin ( $\mathrm{sFt}$ ), since our primary interest was in the effects of ID absent anemia and given the role of sFt in DA metabolism [30, 31, 32]. However, we also used hemoglobin $(\mathrm{Hb})$ as a moderator, given the known demands that brain activity makes on oxygen use.

The purposes of the present study were the following. First, we sought to demonstrate that it is possible to obtain improvements in both brain function (as measured by EEG) and cognition in male and female adolescents in India following consumption of an iron-biofortified staple grain. Second, we sought to determine whether the EEG features related to attention and memory would be those that would mediate the relationship between improvements in blood iron biomarkers and behavioral measures of cognition. Third, we sought to determine whether Hb would be a mediator in the relationship between systemic iron status as measured by sFt and cognition.

\section{Methods}

This study was conducted among a subset of participants in a larger study of the efficacy of a dietary intervention. The parent study involved school children from

[1] The two other general aspects of brain function that can be affected by variations in iron levels are myelination and neurogenesis/synaptogenesis [24, 25, 26, 27]. However, to our knowledge there is no evidence in animal models of changes in whitematter integrity or changes in brain morphology. 
economically disadvantaged families attending boarding schools in Ahmednagar district, Maharashtra, India, a rural community within a two-hour drive of Ahmednagar city. In this double-blind randomized efficacy trial, subjects (school children, 12-16 y) were stratified by hostel of residence and randomly assigned to receive either iron-biofortified pearl millet (BPM; variety ICTP8203, 86 ppm iron, $\mathrm{n}=122$ ) or a popular variety of pearl millet used for comparison (CPM; variety DG9444, 22 ppm iron, $\mathrm{n}=124$ ) from baseline to four months and variety JKBH778 (52 ppm iron) from four to six months. Because of higher-than-expected consumption, the CPM supply was exhausted after four months, and the original CPM variety DG9444 was replaced with variety JKBH778 available in the local market. Post-hoc analysis revealed that JKBH778 had intermediate iron content. All children received approximately 150 to 300 grams (dry) of the selected pearl millet variety daily in the form of a flat bread (bhakri) during lunch and dinner. Consumption was ad libitum. Bhakri was prepared twice daily by seven cooks who used only one type of pearl millet flour, and followed a protocol to standardize bhakri diameter, weight and consistency. Iron status ( $\mathrm{Hb}$, serum ferritin $[\mathrm{sFt}]$, soluble transferrin receptor [sTfR], calculated body iron [BdFe] [33]), inflammation (C-reactive protein [CRP], $\alpha-1$ acid glycoprotein [AGP]), and anthropometric indices were evaluated at enrollment, four months, and six months. This trial was registered at ClinicalTrials.gov, NCT02152150, 02 June 2014. Complete details of the study design and laboratory analyses can be found in $[9,34]$.

\section{Subjects}

Subjects were female and male students (12-16 y) attending a rural boarding school. The school was selected based on the high prevalence of anemia $(>25 \%)$ found in a prescreening survey and on its capacity to support the efficacy trial. Subjects had to be in good health without chronic disease or acute illness, not taking iron supplements or medications that would interfere with iron absorption, residing full-time at the boarding school, and not severely anemic; those with $\mathrm{Hb}$ levels $<85 \mathrm{~g} / \mathrm{L}$ were ineligible and were referred to a physician for follow-up. Of the 288 subjects screened (see Figure 1), 42 were ineligible and 246 were enrolled in the parent feeding trial in September 2011. Anthelminthic treatment (200 mg albendazole) was provided to subjects four weeks before the baseline assessment and at the study midpoint. A subset of 146 subjects was chosen to undergo functional testing -including tests of both physical (not described here) and cognitive function - based on having the lowest ranked screening sFt levels, as it was thought that these individuals would have the greatest potential to benefit from the intervention and demonstrate cognitive change. Of these, a subset of 90 were selected for cognitive testing with concurrent EEG, with equal numbers in each of the two treatment groups; selection was again determined by having the lowest ranked sFt levels; given the low prevalence of inflammation (defined as AGP $>1.0$ or CRP $>5.0$ ), there was no need to correct the sFt measures. The minimum sample size estimated [35] to allow for $80 \%$ power and $\alpha=0.05$ for differences in the amplitudes of event-related potentials, based on EEG data from Otero et al. [10] was 30 per group. After removing subjects for whom usable EEG data (i.e., artifact- and noise-free) was not available at both baseline and endline, the final set of data submitted to analyses was composed of 33 
subjects who consumed the comparison pearl millet and 41 subjects who consumed the biofortified pearl millet.

\section{Cognitive testing with concurrent EEG}

Five cognitive/behavioral tasks - three attention tasks and two memory tasks - assessed aspects of cognition with hypothesized relationship to iron status; procedural details for each of the tasks are presented in the online supporting material (Additional file 1). Testing sessions lasted approximately 60 minutes. DMDX [36] software was used to run all of the tasks, all of which were developed and programmed by MJW. The tasks were presented on a set of Windows-based laptop computers with 36 $\mathrm{cm}$ (diagonal) displays, running at $2.5 \mathrm{GHz}$, with at least $4 \mathrm{~Gb}$ of RAM and at least $320 \mathrm{~Gb}$ of hard disk storage. Stimulus onsets were synchronized to the vertical refresh rate of the monitor and keyboard responses were timed to $\pm 1 \mathrm{~ms}$. Stimuli for the tasks were either grayscale images or white text on a black background (see example stimuli in Additional File 1). The tasks were presented in the following fixed order: 1) The Simple Reaction Time (SRT) task was the most basic task and assessed processing speed absent significant attentional or memory demands. Subjects responded with a single keypress to a symbol that appeared in the center of the screen. 2) The Go/No-Go (GNG) task provided an estimate of the effectiveness of sustained attention and the speed of simple attentional capture. Subjects were required to make a decision about one of two possible stimuli, a vertical or horizontal bar that were randomly assigned to be either a Go or a No-Go stimulus. Subjects were instructed to respond using a button press as quickly as possible when seeing the Go stimulus (which appeared $20 \%$ of the time) and not to respond when seeing the No-Go stimulus (which appeared $80 \%$ of the time). 3) The Attentional Network Task (ANT) was a modified flanker task assessing three distinct functions of attention: alerting (low-level attentional capture), orienting (mid-level spatial selective attention), and conflict (high-level selection or control [37]). On each trial, the participant was presented with either an informative or uninformative cue as to the location of an upcoming test stimulus display (above or below fixation) and was required to press a button to indicate whether a centrally-presented arrow in the display pointed to the left or right, while disregarding flanking elements (congruent, incongruent, or neutral distractors) on either side of the stimulus. Participants were instructed to respond as quickly as possible by pressing one key with their left index finger if the central arrow pointed left and another key with their right index finger if the central arrow pointed right. 4) In the Composite Face Effect (CFE) task, participants learned to identify (using two different buttons) two familiar (famous celebrities; familiarity was confirmed in a pilot test with similarly aged non-participants in the same school) and two unfamiliar faces. They were then shown images in which the top and bottom half of the face could be from the same or different faces and were either aligned or misaligned Participants were instructed to respond only to the cued half of the face and to ignore the other half. 5) Finally, in the Cued Recognition Task (CRT), participants were first shown a set of 24 culturally appropriate pictures, one at a time for three seconds each, to commit to memory. Next, they viewed a set of 48 pictures, 24 from the memorized set and 24 new pictures. Participants were instructed to identify whether the picture was from 
the memorized set or not (i.e. was "old" or "new" to them). The amount of visual information available was varied by covering picture quadrants with black squares such that 50, 75 or 100 percent of any given picture in the second set of 48 was visible. This task was included due to evidence that the integrity of neural circuits supporting recognition memory, particularly those involving the hippocampus, is correlated with the amount of work that can be accomplished during recognition $[38,39]$.

Concurrent EEG data were acquired using a 32-channel geodesic electrode net connected to a 32-channel amplifier (EGI/Philips, Eugene, OR). EEG, as measured at the scalp, reflects the coordinated activity of spatially-adjacent populations of similarly-oriented neurons, propagated through the brain, skull, and skin [40]. Their activity corresponds to the computational operations that are needed to allow a stimulus to be transformed into a response. To the extent that these populations of neurons are effectively carrying out these computations, the amplitude and power of their conjoint activity will be higher, relative to any iron-dependent impairments (e.g., neurotransmitter signaling or oxygen transport). Data were digitized at 1 $\mathrm{KHz}$ during acquisition and down-sampled to $250 \mathrm{~Hz}$ for analyses. Impedances were maintained at $\leq 50 \mathrm{k} \Omega$ during acquisition. Preprocessing for statistical analysis is described in the Supplementary information.

\section{Statistical analyses}

Differences in biomarkers at baseline were analyzed using a 2 (treatment group: $\mathrm{CPM}, \mathrm{BPM}) \times 2$ (sex: female, male) analysis of variance (ANOVAs). Differences in prevalences of biological conditions (e.g., anemia) were analyzed using a $\chi^{2}$ test of association. The first set of analyses were per-protocol analyses of all the dependent measures and took the form of a one-way analysis of covariance (ANCOVA), with treatment group (CPM or BPM) as a between-subjects factor and baseline value of the dependent variable as a covariate, as the magnitude of change can be a function of the starting value [41]. Additional potential covariates included age and sex. Note that each of the dependent measures is a unique assessment of a specific aspect of cognitive functioning, thus there was no need across the set of variables for any corrections for multiple comparisons. A second set of analyses were done to assess the plausibility of the change in iron status as causal in changes in the dependent variables across both treatment groups. These analyses were done by regressing change in the behavioral and EEG variables onto change in the set of iron status biomarkers, testing the hypothesis that adolescents who experienced a greater improvement in iron status would show greater improvement in their behavioral and brain measures. The set of candidate models included a "null" model (intercept only); a full model, in which all allowable independent predictors (iron biomarkers) were included (i.e., sFt and sTfR were not allowed in a model including BdFe); and a model whose form was determined by step-wise model selection procedures to minimize the number of parameters while maximizing $R^{2}$; age and sex were included as potential covariates. We selected a best model using the criteria that the model had to provide a better account than the null model, than any of the competing models (as assessed using the Akaike Information Criterion [42]), and account for at least $10 \%$ of the variance. Age and sex were included as covariates in the final model only if they were shown to be statistically significant. 
The third set of analyses involved estimating a set of mediation models incorporating moderator variables [43] to represent the hypothesis that changes in brain activity mediate the relation between changes in peripheral iron status and changes in behavior. These analyses were performed on three composite ( $Z$-transformed and scaled such that positive values indicate improvement from baseline to endline) behavioral variables: change in low-level attentional capture, change in high-level attentional selection, and change in efficiency of memory retrieval. The attentional capture variable was formed by averaging the $Z$-transformed change in reaction time (RT) in the GNG and the change in RT in the 2-cue condition in the ANT. The attentional selection variable was formed by averaging the $Z$-transformed change in $\mathrm{RT}$ in the spatial cue and inconsistent flanker conditions of the ANT. The memory efficiency variable was formed by averaging the $Z$-transformed change in RT and sensitivity measures in the CFE and the percentage change in capacity (PCC) in the CRT. Three sets of mediating variables were specified for these three composite behavioral variables, based on the regression analyses performed to assess plausibility. The mediating variable for attentional capture was a composite (averaged $Z$-score) of the N1 and P3 amplitudes for the corresponding conditions for the behavioral variables. The mediating variable for attentional selection was a composite of the N1 and P3 amplitudes for the corresponding behavioral conditions. The mediating variable for memory retrieval efficiency was a composite of the N1 and P3 amplitudes and $\gamma$-band power for the corresponding behavioral conditions. The possible predictors/moderators were the normalized (Z-transformed) change in $\mathrm{Hb}, \mathrm{sFt}$, and sTfR, based on the fact that these were the only measures that were identified as significant predictors in the plausibility analyses. These specific variables and composites were selected on the basis of a stepwise procedure which began with all possible variables, and proceeded by removing variables whose parameters were not reliably different from zero (0) and then refitting, and repeating this until we found the smallest set of variables that could account for the greatest amount of variance.

These variables were used to fit candidate mediation models with effect modifiers for each behavioral composite score, using all possible combinations of the EEG composite scores as both mediators and effect modifiers. The final set of variables was determined by first fitting the models with the complete set of variables and then deleting those that did not reach criterion for statistical significance. In addition, a "direct effect only" model and a "scrambled" model were fit to the data for each composite behavioral score. The "direct effect only" model did not include any mediators or effect modifiers, and the "scrambled" model was a model created by rearranging the order of the effects in the best-fitting model. The "best" model met the following criteria: (a) the overall $F$-statistic for the model had to be statistically significant; (b) all component $R^{2}$ values needed to be $\geq 0.10$; (c) all values for model parameters (excluding the intercept) needed to be significantly different from zero; and, (d) based on $R^{2}$ and AIC values, the "best" model had to outperform all alternative models, including the "direct effect only" and "scrambled" models.

\section{Results}

\section{Sample characteristics}

Characteristics of the sample at baseline are presented in Table 1. In terms of the iron biomarkers, the only significant difference was a main effect due to sex for $\mathrm{Hb}$ 
levels, with females overall having lower Hb levels than males. There were no reliable effects due to assignment to treatment condition. There were reliable effects due to sex in terms of prevalence of anemia, IDA, and iron deficiency without anemia (iron deficient non-anemic, IDNA), with higher prevalence in females than in males for anemia and IDA, the opposite ordering for IDNA.

\section{Per-protocol analyses}

Results of the per-protocol analyses of the endline blood iron biomarkers and behavioral variables are presented in Table 2 . For the blood iron biomarkers, baseline value was statistically significant for all of the variables, and condition was statistically significant for all of the variables except $\mathrm{Hb}$. Sex was the only one of the two potential covariates that was statistically significant, and that was true only for sFt and BdFe. For the behavioral variables, baseline value was statistically significant for only a subset of the variables, and it accounted for a relatively small proportion of the total variance $(\max =0.26$, mean $=0.06)$. Treatment condition was statistically significant for 14 of the 21 variables and accounted for a much larger proportion of the total variance $(\max =0.59$, mean $=0.18)$ than was the case for baseline value. In each case in which treatment condition was significant, the amount of improvement was larger for the group consuming the BPM than for the group consuming the CPM grain. Age was statistically significant as a covariate for eight of the variables; sex was statistically significant as a covariate for only one of the variables.

Results of the per-protocol analyses of the EEG variables are presented in Table 3. Baseline value was statistically significant for 18 of the 60 variables and overall accounted for a relatively small proportion of the variance $(\max =0.72$, mean $=$ 0.05). Treatment condition was statistically significant for 23 of the 60 variables and accounted for a much larger proportion of the total variance $(\max =0.96$, mean $=$ 0.21) than was true for baseline value. In each case for which treatment condition was significant, the amount of improvement was greater for those in the BPM group than for those in the CPM group. Finally, age and sex were statistically significant covariates for only two of the variables.

A summary of the amount of change observed for all of the dependent variables is plotted as the difference between the normalized (Z-unit) change for the BPM group and normalized change for the CPM group in Figures 2 and 3; Figure 2 plots the results for the attentional variables and Figure 3 plots the results for the mnemonic variables. Note that, in all cases for all variables, change was greatest for those consuming the BPM (as indicated by all of the difference scores being $>0$. Also note that, relative to the smallest amount of positive change observed for the blood variables, the magnitude of the positive difference was larger for the majority of the behavioral and EEG variables.

\section{Plausibility analyses}

The results of the regression analyses performed to assess the plausibility that changes observed in the behavioral and the EEG variables were related to changes in blood parameters are reported in Table 4. For the behavioral variables, all of the tasks except for the SRT had at least one variable for which the regression met our 
criteria for sufficiency. Of the 16 behavioral variables for which this was true, 13 of the cases involved $\mathrm{sFt}$ or BdFe as the best predictor, with two of the remaining cases having $\mathrm{Hb}$ as the best predictor and one having sTfR as the best predictor. ${ }^{[2]}$ For those variables for which a model met our criteria, the average proportion of variance accounted for was 0.17 . With respect to the EEG variables, all of the tasks had at least one variable for which a model met our sufficiency criteria. In those cases, either sFt or BdFe were selected as the best predictor in all but one case, in which $\mathrm{Hb}$ was selected as the best predictor. For those variables that had a model that met our sufficiency criteria, the average proportion of variance accounted for was 0.16 .

\section{Mediation analyses}

The common form for the selected mediation models for each of the three composite variables is presented in Figure 4, and the estimated parameters for each model are presented in Table 5. For all three composite variables, change in sFt was selected as the primary predictor and change in $\mathrm{Hb}$ was selected as an effect modifying variable. Specifically, the effect of a change in sFt on change in behavior was mediated by a change in brain activity. In addition, both the direct relationship between change in $\mathrm{sFt}$ and change in behavior and the relationship between change in sFt and change in brain activity was modified by change in $\mathrm{Hb}$. On average, the predicted change in the mediating variables accounted for $36 \%$ of the total variance and the predicted change in the outcomes accounted for $21 \%$ of the variance.

\section{Discussion}

The literature on the functional effects of ID and IDA has an increasing number of examples documenting that ID and IDA extract significant costs in terms of cognition $[44,45,8,46,12,6]$. The present study is among the first to depart from a descriptive approach to move towards tests of hypotheses regarding mechanisms. We focused on controlled attention and memory, as these are functions that have been shown to be dependent on the integrity of the dopaminergic system, and work in animal models have pointed to significant effects of ID/IDA on the dopaminergic system. In addition, the study is among the first to both collect simultaneous behavioral and EEG data and to explicitly model the mediating role that changes in brain function have in relating changes in blood iron biomarkers to changes in behavior. Finally, the present study is among the first to explicitly model the hypothesis that changes in oxygen transport capacity (as measured by changes in $\mathrm{Hb}$ ) work in conjunction with changes in neurotransmitters (as suggested by changes in sFt).

We demonstrated that changes in blood iron biomarkers were associated with reliable changes in both behavioral and EEG features associated with a variety of aspects of attention. These included changes in power in the $\alpha$ and $\gamma$ frequency bands and, most regularly, the amplitude of the N1 component. This suggests the

${ }^{[2]}$ Note that sFt and sTfR are combined in calculating BdFe, and each assess different aspects of bodyn iron status (liver store vs. tissue iron demand, respectively). Consequently, BdFe as a composite measure is a more general indicator of body iron status. 
plausibility of the hypothesis that ID produces negative changes in dopaminergic regulation, particularly in basal ganglia, and that repletion corrects this, with the outcome measurable as a function of the output of basal ganglia circuits to cortex, and replicates a similar set of results obtained with a population of college-aged women consuming an iron biofortified bean [38].

We also demonstrated, by way of statistical modeling of the relationships among the three classes of variables (blood iron biomarkers, ERP data, and behavioral measures), that the negative effects of iron deficiency with and without anemia on cognition may also be a function of deficits in oxygen transport. A sizable minority $(35 \%)$ of the participants in this study had Hb levels below the criterion for anemia. Consequently, it is perhaps not surprising that the statistical modeling of the relationships among the blood, brain, and behavioral variables showed that change in $\mathrm{Hb}$ needed to be included as a positively-signed moderator in order to provide the best description of the data, even though $\mathrm{Hb}$ played a minor role in the plausibility analyses. This suggests that a comprehensive understanding of the mechanisms by which ID/IDA impairs brain function needs to consider effects both at the level of neurotransmitter function and at the level of oxygen transport.

All of these results were obtained in the context of the first study to document the effectiveness of providing an iron-biofortified grain as a regular dietary component on the iron status of both male and female adolescents. This pattern of results conceptually replicates previous work [38], in which we demonstrated that consumption of a bean, biofortified for increased iron, was capable of producing improvements in blood iron biomarkers, behavioral measures of cognition, and EEG measures of brain function in college-aged women in Rwanda. This suggests that the effectiveness of using a dietary intervention to address ID/IDA extends beyond benefits to the body to benefits to brain function and cognition, and is consistent with the outcomes of other studies that have examined the effects of iron depletion and repletion in children, adolescents, and women of reproductive age [6, 10, 11, 45, 47]. It also highlights the importance of biofortification as an intervention, in that it is low in dosage, targeted to the most needy, sustainable, and efficacious at numerous levels of measurement.

The strengths of the present effort include the fact that it was conducted as a randomized, double-blind controlled study. Furthermore, as it concerned the effects of the dietary intervention on functional outcomes that are determined by brain function, it incorporated measures of blood, brain function, and behavior. Additionally, it used cognitive measures that have a long history and rich literature in laboratory work on basic cognition and that were selected based on their dependence on brain regions that are differentially dependent on iron. Finally, it applied statistical methods that allow for assessment of the mediation hypothesis and that are flexible enough to explicitly include hypothesized modifying influences.

The weaknesses of the effort include the fact that the conditions under which the EEG was recorded were far from ideal. Recording was not done in electromagneticallyshielded rooms, and the power source for recording was somewhat unreliable and electrically noisy. As a consequence, even with extensive pre-processing, there was still loss of data. In addition, the EEG data as collected cannot speak to the range of possible mechanisms supporting the changes in the behavioral measures. Although 
the mediation models provide suggestive evidence of a causal link, they can only be weakly interpreted as evidence for causality. Finally, data were not available on menstrual cycle timing in female participants, and this may have influenced blood biomarker levels; however, randomization should have addressed this concern.

\section{Conclusion}

In sum, we have provided evidence that consumption of a iron-biofortified pearl millet for six months leads to improvements in multiple measures of iron status, brain function, and cognition. These results add to the increasing evidence that biofortification is an effective method for addressing micronutrient deficiencies and to the evidence that iron repletion by way of a nutritional intervention other than supplementation has measurable and important effects on brain and cognitive function. Furthermore, the inclusion of measures of brain function provide evidence important in motivating future work on the brain mechanisms responsible for supporting changes in cognition and their inherent plasticity.

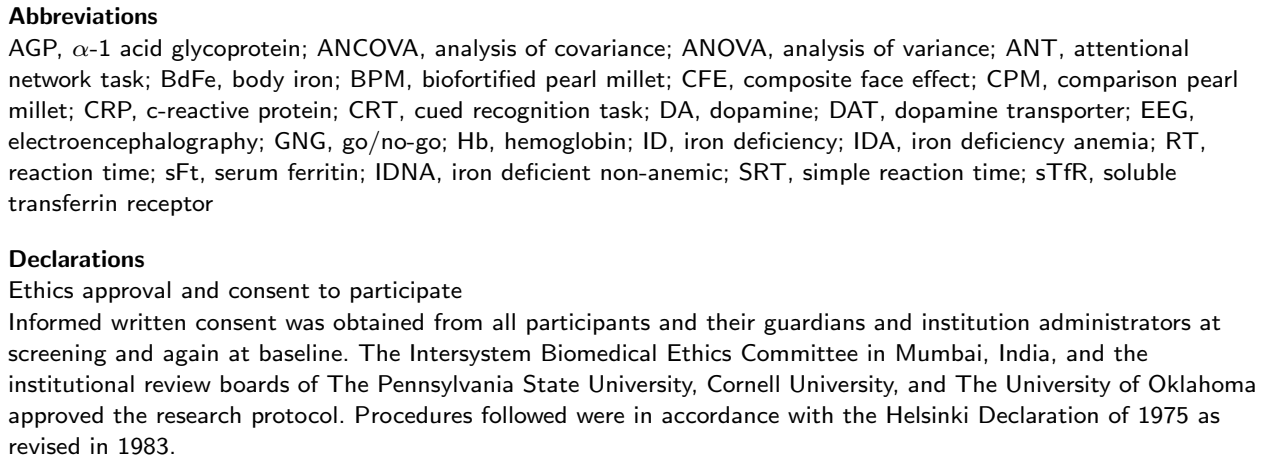


Author details

${ }^{1}$ Department of Psychology, Cellular and Behavioral Neurobiology, The University of Oklahoma, Norman, OK, USA.

${ }^{2}$ Division of Nutritional Sciences, Cornell University, Ithaca, NY, USA. ${ }^{3}$ Department of Nutrition Science, Purdue University, West Lafayette, IN, USA. ${ }^{4}$ Poverty Health and Nutrition Division, International Food Policy Research Institute, Washington, DC, USA. ${ }^{5}$ HarvestPlus, International Food Policy Research Institute, Washington, DC, USA

References

1. Stevens, G.A., Finucane, M.M., De-Regil, L.M., Paciorek, C.J., Flaxman, S.R., F. Branca, F., Group., N.I.M.S Global, regional, and national trends in haemoglobin concentration and prevalence of total and severe anaemia in children and pregnant and non-pregnant women for 1995-2011: A Systematic analysis of population-representative data. The Lancet Global Health 1(1), 16-25 (2013)

2. Pasricha, S.-R., Low, M., Thompson, J., Farrell, A., De-Regil, L.-M.: Iron supplementation benefits physical performance in women of reproductive age: a systematic review and meta-analysis. The Journal of nutrition 144(6), 906-914 (2014)

3. Haas, J.D.: The effects of iron deficiency on physical performance. Food and Nutrition Board, Institute of Medicine. The National Academies Press, Washington, DC, 451-461 (2006)

4. Haas, J.D., Brownlie, T.: Iron deficiency and reduced work capacity: A critical review of the research to determine a causal relationship. Journal of Nutrition 131, 676-688 (2001)

5. Li, R., Chen, X., Yan, H., Deurenberg, P., Garby, L., Hautvast, J.: Functional consequences of iron supplementation in iron-deficient female cotton mill workers in beijing, china. The American Journal of Clinical Nutrition 59(4), 908-913 (1994)

6. Wenger, M.J., Murray-Kolb, L.E., Nevins, J.E.H., Venkatramanan, S., Reinhart, G.A., Wesley, A., Haas, J.D.: Consumption of a double-fortified salt affects perceptual, attentional, and mnemonic functioning in women in a randomized controlled trial in india. The Journal of Nutrition 147(12), 2297-2308 (2017)

7. Wenger, M.J., DellaValle, D.M., Muuray-Kolb, L.E., Haas, J.D.: Effect of iron deficiency on simultaneous measures of behavior, brain activity, and energy expenditure in the performance of a cognitive task. Nutritional Neuroscience 22(3), 196-206 (2017)

8. Murray-Kolb, L.E., Wenger, M.J., Scott, S.P., Rhoten, S.E., Lung'aho, M.G., Haas, J.D.: Consumption of iron-biofortified beans positively affects cognitive performance in 18- to 27 -year-old Rwandan female college students in an 18-week randomized controlled efficacy trial. Journal of Nutrition 147(11), 2109-2117 (2017)

9. Scott, S.P., Murray-Kolb, L.E., Wenger, S.A. M. J .and Udipi, Ghugre, P.S., Boy, E., Haas, J.D.: Cognitive performance in indian school-going adolescents is positively affected by consumption of iron-biofortified pear millet: A 6-month randomized controlled efficacy trial. The Journal of Nutrition 148(9), 1462-1471 (2018)

10. Otero, G.A., Pliego-Rivero, F.B., Contreras, G., Ricardo, J., Fernández, T.: Iron supplementation brings up a lacking P300 in iron deficient children. Clinical Neurophysiology 115, 2259-2266 (2004)

11. Otero, G.A., Fernández, T., Pliego-Rivero, F.B., Mendieta, G.G.: Iron therapy substantially restores qeeg maturational lag among iron-deficient anemic infants. Nutritional Neuroscience 22(5), 363-372 (2019)

12. Scott, S.P., De Souza, M.J., Koehler, K., Murray-Kolb, L.E.: Combined iron deficiency and low aerobic fitness doubly burden academic performance among women attending university. The Journal of Nutrition 147(1), 104-109 (2017)

13. Unger, E.L., Wiesinger, J.A., Hao, L., Beard, J.L.: Dopamine D2 receptor expression is altered by changes in cellular iron levels in PC12 cells and rat brain tissue. The Journal of Nutrition 138(12), 2487-2494 (2009)

14. Unger, E.L., Hurst, A.R., Georgieff, M.K., Schallert, T., Rao, R., Connor, J.R., Kaciroti, N., Lozoff, B., Felt, B.: Behavior and monoamine deficits in prenatal and perinatal iron deficiency are not corrected by early postnatal moderate-iron or high-iron diets in rats. The Journal of Nutrition 142(11), 2040-2049 (2012)

15. Unger, E.L., Bianco, L.E., Jones, B.C., Allen, R.P., Earley, C.J.: Low brain iron effects and reversibility on striatal dopamine dynamics. Experimental Neurology 261, 462-468 (2014)

16. Lozoff, B.: Early iron deficiency has brain and behavior effects consistent with dopaminergic dysfunction. The Journal of Nutrition 141(4), 740-746 (2011)

17. Karson, C.N.: Spontaneous eye-blink rates and dopaminergic systems. Brain 106(3), 643-653 (1983)

18. Broadway, J.M., Frank, M.J., Cavanagh, J.F.: Dopamine D2 agonist affects visuospatial working memory distractor interference depending on individual differences in baseline working memory span. Cognitive, Affective, and Behavioral Neuroscience 18(3), 509-520 (2018)

19. Cools, R.: Role of dopamine in the motivational and cognitive control of behavior. The Neuroscientist 14(4), 381-395 (2008)

20. Cools, R., D'Esposito, M.: Inverted-U-shaped dopamine actions on human working memory and cognitive control. Biological psychiatry 69(12), 113-125 (2011)

21. Fox, P.T., Raichle, M.E., Mintun, M.A., Dence, C.: Nonoxidative glucose consumption during focal physiologic neural activity. Science 241(4864), 462-464 (1988)

22. Fox, P.T., Raichle, M.E.: Focal physiological uncoupling of cerebral blood flow and oxidative metabolism during somatosensory stimulation in human subjects. Proceedings of the National Academy of Science USA 83(4), 1140-1144 (1986)

23. Raichle, M.E., Gusnard, D.A.: Appraising the brain's energy budget. Proceedings of the National Academy of Science USA 99(16), 10237-10239 (2002)

24. Burhans, M.S., Dailey, C., Wiesinger, J., Murray-Kolb, L.E., Jones, B.C., Beard, J.L.: Iron deficiency affects acoustic startle response and latency, but not prepulse inhibition in young adult rats. Physiology \& behavior 87(5), 917-924 (2006)

25. Erikson, K.M., Jones, B.C., Hess, E.J., Zhang, Q., Beard, J.L.: Iron deficiency decreases dopamine d 1 and d 2 receptors in rat brain. Pharmacology Biochemistry and Behavior 69(3), 409-418 (2001)

26. Nelson, C., Erikson, K., Piñero, D.J., Beard, J.L.: In vivo dopamine metabolism is altered in iron-deficient anemic rats. The Journal of nutrition 127(12), 2282-2288 (1997) 
27. Masini, A., Trenti, T., Caramazza, I., Predieri, G., Gallesi, D., Ceccarelli, D.: Dietary iron deficiency in the rat. II. Recovery from energy metabolism derangement of the hepatic tissue by iron therapy. Biochimica et Biophysica Acta (BBA)-Bioenergetics 1188(1-2), 53-57 (1994)

28. Angelidis, A., Hagenaars, M., van Son, D., van der Does, W., Putman, P.: Do not look away! spontaneous frontal eeg theta/beta ratio as a marker for cognitive control over attention to mild and high threat. Biological Psychology 135, 8-17 (2018)

29. Shestyuk, A.Y., Kasinathan, K., Karapoondinott, V., Knight, R.T., Gurumoorthy, R.: Individual EEG measures of attention, memory, and motivation predict population level tv viewership and twitter engagement. PloS one 14(3) (2019)

30. Snyder, A.M., Connor, J.R.: Iron, the substantia nigra and related neurological disorders. Biochimica et Biophysica Acta (BBA)-General Subjects 1790(7), 606-614 (2009)

31. Larsen, B., Olafsson, V., Calabro, F., Laymon, C., Tervo-Clemmens, B., Campbell, E., Minhas, D., Montez, D., Price, J., Luna, B.: Maturation of the human striatal dopamine system revealed by pet and quantitative mri. Nature Communications 11(1), 1-10 (2020)

32. Ward, R.J., Zucca, F.A., Duyn, J.H., Crichton, R.R., Zecca, L.: The role of iron in brain ageing and neurodegenerative disorders. The Lancet Neurology 13(10), 1045-1060 (2014)

33. Cook, J.D., Flowers, C.H., Skikne, B.S.: The quantitative assessment of body iron. Blood 101, 3359-3364 (2003)

34. Finkelstein, J.L., Mehta, S., Udipi, S.A., Ghugre, P.S., Luna, S.V., Wenger, M.J., Murray-Kolb, L.E., Przybyszewski, E.M., Haas, J.D.: A randomized trial of iron-biofortified pearl millet in school children in india. The Journal of nutrition 145(7), 1576-1581 (2015)

35. Zar, J.H.: Biostatistical Analysis, 4th edn. Prentice Hall, Upper Saddle River, NJ (1999)

36. Forster, K.I., Forster, J.C.: DMDX: A Windows display program with millisecond accuracy. Behavioral Research Methods, Instruments, and Computers 35, 116-124 (2003)

37. Fan, J., McCandliss, B.D., Sommer, T., Raz, A., Posner, M.I.: Testing the efficiency and independence of attentional networks. Journal of Cognitive Neuroscience 14(3), 340-347 (2002)

38. Wenger, M.J., Rhoten, S.E., Murray-Kolb, L.E., Scott, S.P., Boy, E., Gahutu, J.-B., Haas, J.D.: Changes in iron status are related to changes in brain activity and behavior in Rwandan female university students: Results from a randomized controlled efficacy trial involving iron-biofortified beans. Journal of Nutrition 149(4), 687-697 (2019)

39. Wenger, M.J., Negash, S., Petersen, R.C., Petersen, L.: Modeling and esimating recall processing capacity: Sensitivity and diagnostic utility in application to mild cognitive impairment. Journal of Mathematical Psychology 54, 73-89 (2010)

40. Nunez, P.L., Srinivasan, R.: Electric Fields of the Brain: The Neurophysics of EEG, 2nd edn. Oxford, Oxford (2006)

41. Fairchild, M.W., Haas, J.D., Habicht, J.-P.: Iron deficiency and behavior: criteria for testing causality. The American Journal of Clinical Nutrition 50(3), 566-574 (1989)

42. Akaike, $\mathbf{H}$.: Information measures and model selection. Bulletin of the International Statistical Institute $\mathbf{5 0}$ 277-290 (1983)

43. Hayes, A.F.: Introduction to Mediation, Moderation, and Conditional Process Analysis: A Regression-based Approach, 2nd edn. Guilford Press, New York (2018)

44. Murray-Kolb, L.E., Beard, J.L.: Iron treatment normalizes cognitive functioning in young women. American Journal of Clinical Nutrition 85, 778-787 (2007)

45. Otero, G., Pliego-Rivero, F., Porcayo-Mercado, R.: Working memory impairment and recovery in iron deficient children. Clinical Neurophysiology 115, 2259-2266 (2008)

46. Scott, S.P., Murray-Kolb, L.E.: Iron status is associated with performance on executive functioning tasks in nonanemic young women. The Journal of Nutrition 146(1), 30-37 (2016)

47. Otero, G.A., Aguirre, D.M., Porcayo, R., Fernandez, T.: Psychological and electroencephalographic study in school children with iron deficiency. International Journal of Neuroscience 99(1-4), 113-121 (1999)

Figures

Figure 1 CONSORT diagram for the study.

Figure 2 Magnitude of the difference in the normalized amount of change observed for those consuming the biofortified pearl millet (BPM) and those consuming the control pearl millet (CPM), on each of the blood, behavior, and EEG variables for the attention tasks. The reference lines denote the smallest amount of change observed for the blood biomarkers. 
Figure 3 Magnitude of the difference in the normalized amount of change observed for those consuming the biofortified pearl millet (BPM) and those consuming the control pearl millet (CPM), on each of the blood, behavior, and EEG variables for the memory tasks. The reference lines denote the smallest amount of change observed for the blood biomarkers.

Figure 4 Common form for the best-fitting mediation models with effect modifiers. Change refers to change from baseline to endline. In all cases (see Table 5, all parameters were positive.

Tables 
Table 1 Baseline characteristics of the sample and comparisons across conditions. Means (standard deviations) are reported for males and females in both treatment conditions, along with F-statistics for the two-way ANOVA and $\chi^{2}$ statistics for the tests of association. Note: $+=0.05<=p<0.10, *=p<0.05, * *=p<0.01, \mathrm{CPM}=$ control pearl millet, $\mathrm{BPM}=$ biofortified pearl millet.

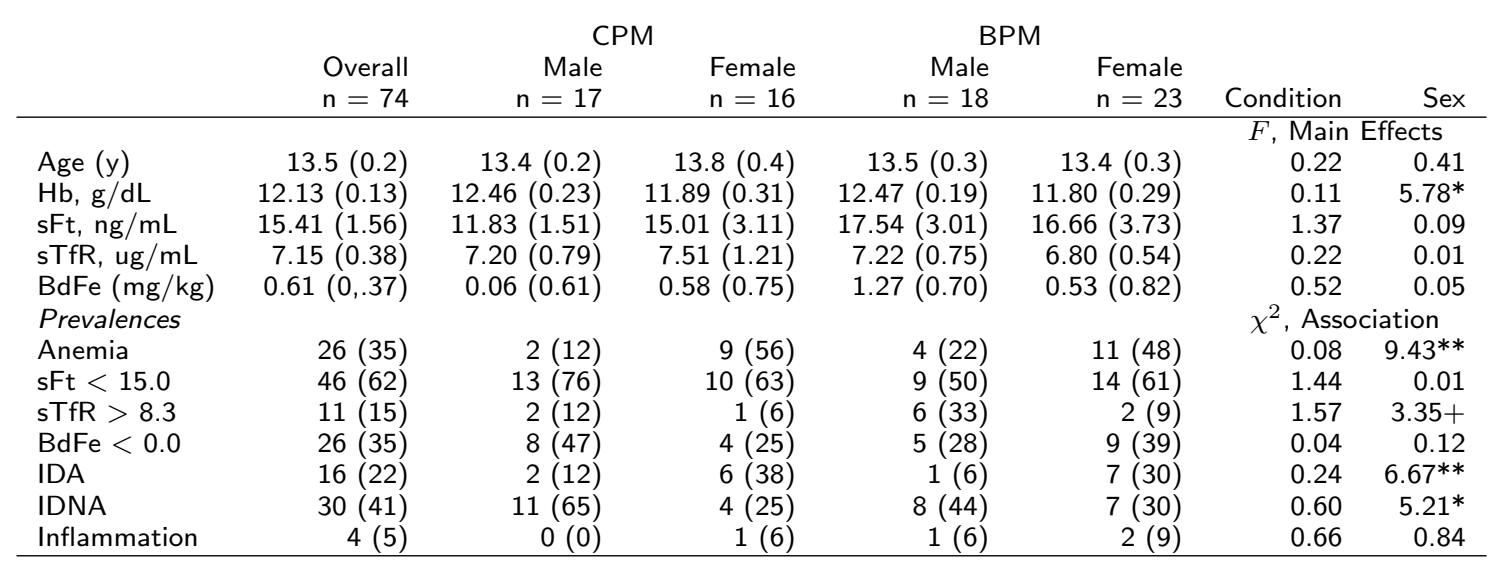


Table 2: Per-protocol analyses of the blood iron biomarkers and behavioral data. Note: $+=0.05 \leq p<0.10, *=p<0.05, * *=p<0.01, * * *=p<$ 0.001 .

\begin{tabular}{|c|c|c|c|c|c|c|c|}
\hline \multirow[b]{2}{*}{ Task } & \multirow[b]{2}{*}{ EL Variable } & \multicolumn{3}{|c|}{ Effect of BL Value } & \multicolumn{2}{|c|}{ Effect of Treatment Condition } & \multirow[b]{2}{*}{ Covariates } \\
\hline & & $F$ & MSE & partial $\eta^{2}$ & $F$ & partial $\eta^{2}$ & \\
\hline \multicolumn{8}{|c|}{ Blood variables } \\
\hline & $\mathrm{Hb}$ & $30.10 * * *$ & 0.93 & 0.3 & 2.43 & 0.03 & \\
\hline & $\mathrm{sFt}$ & $10.83^{* *}$ & 104.26 & 0.13 & $7.71^{* *}$ & 0.10 & sex \\
\hline & sTfR & $6.47^{*}$ & 0.12 & 0.08 & $7.90 * *$ & 0.10 & \\
\hline & $\mathrm{BdFe}$ & $68.09 * * *$ & 4.93 & 0.49 & $3.46+$ & 0.05 & sex \\
\hline \multicolumn{8}{|c|}{ Behavioral variables } \\
\hline SRT & SRT: median RT & 0.19 & 11194 & 0.02 & 1.63 & 0.02 & \\
\hline GNG & GNG: median RT & 0.23 & 3474 & 0.00 & $11.24 * *$ & 0.14 & age \\
\hline \multirow[t]{9}{*}{ ANT } & ANT: RT, 0 cues & $20.58 * * *$ & 3824 & 0.23 & 1.25 & 0.02 & age \\
\hline & ANT: RT, 2 cues & 1.66 & 6063 & 0.02 & $11.37^{* *}$ & 0.14 & age, sex \\
\hline & ANT: RT alerting & $3.09+$ & 2568 & 0.04 & $36.37 * * *$ & 0.34 & sex \\
\hline & ANT: RT, center cues & $20.74 * * *$ & 3873 & 0.23 & 1.45 & 0.02 & \\
\hline & ANT: RT, spatial cues & 0.42 & 4438 & 0.01 & $9.52 * *$ & 0.25 & age \\
\hline & ANT: $R T$, orienting & 0.1 & 1796 & 0.00 & $48.21^{* * *}$ & 0.53 & \\
\hline & ANT: RT, consistent flankers & $23.79 * * *$ & 5347 & 0.25 & 4.70* & 0.17 & \\
\hline & ANT: RT, inconsistent flankers & $11.61^{* *}$ & 7710 & 0.26 & $24.47^{* * *}$ & 0.39 & \\
\hline & ANT: RT, conflict & 0.51 & 5094 & 0.01 & $62.38 * * *$ & 0.59 & \\
\hline \multirow[t]{5}{*}{ CFE } & CFE: RT & 0.09 & 6281 & 0.00 & $5.46^{*}$ & 0.07 & \\
\hline & CFE: Hit rate & 1.27 & 0.01 & 0.02 & $2.98+$ & 0.04 & \\
\hline & CFE: FA rate & 0.11 & 0.02 & 0.00 & 1.88 & 0.03 & \\
\hline & CFE: sensitivity & 1.36 & 0.22 & 0.02 & $9.04 * * *$ & 0.26 & \\
\hline & CFE: bias & 2.09 & 0.09 & 0.03 & 2.03 & 0.03 & \\
\hline \multirow[t]{4}{*}{ CRT } & CRT: Sensitivity & 0.34 & 0.83 & 0.00 & $7.41^{* *}$ & 0.10 & \\
\hline & CRT: Bias & 0.40 & 0.20 & 0.01 & 1.37 & 0.02 & \\
\hline & CRT: New items & 1.90 & 26282 & 0.03 & 0.62 & 0.09 & age \\
\hline & CRT: Old items & 3.42 & 10483 & 0.05 & $11.33 * *$ & 0.15 & age \\
\hline
\end{tabular}


Table 2: Per-protocol analyses of the blood iron biomarkers and behavioral

data. Note: $+=0.05 \leq p<0.10, *=p<0.05, * *=p<0.01, * * *=p<$

$$
0.001 \text {. }
$$

Task

EL Variable CRT: PCC

Effect of BL Value

F MSE partial $\eta^{2}$
Effect of Treatment Condition 0.01

partial $r$

0.35 Covariates


Table 3: Per-protocol analyses of the EEG data. Note: $+=0.05 \leq p<$

$0.10, *=p<0.05, * *=p<0.01, * * *=p<0.001$.

\begin{tabular}{|c|c|c|c|c|c|c|c|}
\hline \multirow[b]{2}{*}{ Task } & \multirow[b]{2}{*}{ EL Variable } & \multicolumn{3}{|c|}{ Effect of BL Value } & \multicolumn{2}{|c|}{ Effect of Treatment Condition } & \multirow[b]{2}{*}{ Covariates } \\
\hline & & $F$ & MSE & partial $\eta^{2}$ & $F$ & partial $\eta^{2}$ & \\
\hline \multirow[t]{4}{*}{ SRT } & N1 amplitude & 0.18 & 2.09 & 0.00 & $85.67 * * *$ & 0.72 & \\
\hline & P3 amplitude & 0.12 & 1.39 & 0.00 & $4.57^{*}$ & 0.06 & \\
\hline & alpha power & 0.00 & 0.0001 & 0.00 & 0.97 & 0.01 & \\
\hline & gamma power & 0.00 & 0.0002 & 0.00 & 1.94 & 0.03 & \\
\hline \multirow[t]{4}{*}{ GNG } & N1 amplitude & 1.43 & 1.07 & 0.02 & $5.88^{*}$ & 0.08 & \\
\hline & P2/P3 amplitude & 0.02 & 1.01 & 0.00 & 1.93 & 0.03 & \\
\hline & alpha power & 1.36 & 176.52 & 0.02 & 0.02 & 0.00 & \\
\hline & gamma power & 3.97 & 1.33 & 0.05 & 2.75 & 0.04 & age \\
\hline \multirow[t]{19}{*}{ ANT } & 0 cues: N1 amplitude & 2.70 & 2.49 & 0.04 & 0.70 & 0.01 & \\
\hline & 0 cues: P3 amplitude & 0.00 & 0.68 & 0.00 & $2.84+$ & 0.04 & \\
\hline & 0 cues: alpha power & $181.78 * * *$ & 6.07 & 0.73 & 0.79 & 0.01 & \\
\hline & 0 cues: gamma power & $61.60 * * *$ & 11.39 & 0.48 & 0.38 & 0.01 & \\
\hline & 2 cues: N1 amplitude & $30.56 * * *$ & 5.42 & 0.31 & $6.51^{* *}$ & 0.09 & \\
\hline & 2 cues: P3 amplitude & 0.00 & 0.68 & 0.00 & $4.57^{*}$ & 0.06 & \\
\hline & 2 cues: alpha power & $173.81^{* * *}$ & 3.41 & 0.85 & 0.02 & 0.00 & \\
\hline & 2 cues: gamma power & $41.99 * * *$ & 13.30 & 0.38 & 1.65 & 0.02 & \\
\hline & Alerting: N1 amplitude & $33.71^{* * *}$ & 3.29 & 0.33 & $7.37 * *$ & 0.10 & \\
\hline & Alerting: P3 amplitude & 0.15 & 0.15 & 0.00 & 0.90 & 0.01 & \\
\hline & Alerting: alpha power & $6.29 *$ & 2.00 & 0.08 & 0.19 & 0.00 & \\
\hline & Alerting: gamma power & 1.47 & 7.35 & 0.02 & 0.07 & 0.00 & \\
\hline & Center cues: N1 amplitude & $26.96 * * *$ & 0.12 & 0.28 & 1.23 & 0.02 & \\
\hline & Center cues: P3 amplitude & 0.80 & 0.32 & 0.01 & 1.16 & 0.02 & \\
\hline & Center cues: alpha power & 1.03 & 21.76 & 0.01 & 0.03 & 0.00 & \\
\hline & Center cues: gamma power & 1.46 & 23.96 & 0.02 & $3.76+$ & 0.05 & \\
\hline & Spatial cues: N1 amplitude & 0.04 & 1.27 & 0.00 & $6.84 *$ & 0.09 & \\
\hline & Spatial cues: P3 amplitude & 0.21 & 0.93 & 0.00 & $13.82 * * *$ & 0.17 & \\
\hline & Spatial cues: alpha power & 0.50 & 24.62 & 0.01 & 0.15 & 0.00 & \\
\hline
\end{tabular}


Table 3: Per-protocol analyses of the EEG data. Note: $+=0.05 \leq p<$

$0.10, *=p<0.05, * *=p<0.01, * * *=p<0.001$.

\begin{tabular}{|c|c|c|c|c|c|c|c|}
\hline \multirow[b]{2}{*}{ Task } & \multirow[b]{2}{*}{ EL Variable } & \multicolumn{3}{|c|}{ Effect of BL Value } & \multicolumn{2}{|c|}{ Effect of Treatment Condition } & \multirow[b]{2}{*}{ Covariates } \\
\hline & & $F$ & MSE & partial $\eta^{2}$ & $F$ & partial $\eta^{2}$ & \\
\hline & Spatial cues: gamma power & 0.10 & 26.80 & 0.00 & 1.67 & 0.02 & \\
\hline & Orienting: N1 amplitude & 0.71 & 1.21 & 0.01 & $6.3^{*}$ & 0.09 & \\
\hline & Orienting: P3 amplitude & 0.15 & 0.70 & 0.00 & $12.90 * * *$ & 0.16 & \\
\hline & Orienting: alpha power & 0.35 & 3.92 & 0.01 & 0.98 & 0.01 & \\
\hline & Orienting: gamma power & 1.70 & 5.98 & 0.02 & $3.48+$ & 0.05 & \\
\hline & Congruent flankers: N1 amplitude & 0.19 & 0.39 & 0.00 & 0.05 & 0.00 & \\
\hline & Congruent flankers: P3 amplitude & 0.19 & 0.68 & 0.00 & 0.10 & 0.00 & \\
\hline & Congruent flankers: alpha power & $2.97+$ & 26.27 & 0.04 & 0.06 & 0.00 & \\
\hline & Congruent flankers: gamma power & 1.27 & 24.27 & 0.02 & $5.67^{*}$ & 0.08 & \\
\hline & Incongruent flankers: N1 amplitude & 0.01 & 0.67 & 0.00 & $7.21^{* *}$ & 0.10 & \\
\hline & Incongruent flankers: P3 amplitude & 0.07 & 4.76 & 0.00 & $7.51^{* *}$ & 0.10 & \\
\hline & Incongruent flankers: alpha power & 0.93 & 22.06 & 0.01 & 0.43 & 0.00 & \\
\hline & Incongruent flankers: gamma power & 0.04 & 22.59 & 0.00 & 1.74 & 0.02 & \\
\hline & Conflict: N1 amplitude & 2.32 & 0.66 & 0.03 & $6.60 *$ & 0.09 & \\
\hline & Conflict: P3 amplitude & $4.76^{*}$ & 4.53 & 0.07 & $5.05^{*}$ & 0.07 & \\
\hline & Conflict: alpha power & 0.08 & 9.00 & 0.00 & 0.70 & 0.01 & \\
\hline & Conflict: gamma power & 0.05 & 12.19 & 0.00 & $3.78+$ & 0.05 & \\
\hline \multirow[t]{4}{*}{ CFE } & Interaction contrast: N1 amplitude & $2.98+$ & 3.93 & 0.04 & $3.92 *$ & 0.06 & age \\
\hline & Interaction contrast: P3 amplitude & $89.44 * * *$ & 0.43 & 0.57 & 0.54 & 0.00 & \\
\hline & Interaction contrast: alpha power & $21.77^{* * *}$ & 2.54 & 0.24 & 0.04 & 0.00 & \\
\hline & Interaction contrast: gamma power & 1.00 & 4.74 & 0.01 & 1.78 & 0.01 & \\
\hline \multirow[t]{7}{*}{ CRT } & New items, 4 cues, N1 amplitude & $51.52 * * *$ & 0.24 & 0.91 & 0.15 & 0.00 & \\
\hline & New items, 4 cues, P3 amplitude & $33.84 * * *$ & 0.33 & 0.84 & 1.52 & 0.03 & \\
\hline & Old items, 4 cues, N1 amplitude & $47.59 * * *$ & 0.27 & 0.88 & 0.01 & 0.00 & sex \\
\hline & Old items, 4 cues, P3 amplitude & $18.98 * * *$ & 3.71 & 0.26 & $6.01^{*}$ & 0.10 & sex \\
\hline & N1 amplitude slope, old items & $29.92 * * *$ & 0.16 & 0.80 & $2.76+$ & 0.05 & \\
\hline & P3 amplitude slope, old items & $17.88^{* * *}$ & 1.06 & 0.25 & $2.92+$ & 0.10 & \\
\hline & New items, 4 cues, alpha power & $66.49 * * *$ & 5.47 & 0.93 & 0.00 & 0.00 & \\
\hline
\end{tabular}


Table 3: Per-protocol analyses of the EEG data. Note: $+=0.05 \leq p<$

$0.10, *=p<0.05, * *=p<0.01, * * *=p<0.001$.

\begin{tabular}{llrrrrr} 
& & \multicolumn{3}{c}{ Effect of BL Value } & \multicolumn{2}{c}{ Effect of Treatment Condition } \\
Task & $F$ & MSE & partial $\eta^{2}$ & $F$ & 0.08 \\
partial $\eta^{2}$ & Covariates \\
\hline New items, 4 cues, gamma power & $55.33^{* * *}$ & 2.55 & 0.87 & $3.84^{*}$ & 0.00 \\
Old items, 4 cues, alpha power & $31.30^{* * *}$ & 3.02 & 0.96 & 0.16 & 0.07 \\
Old items, 4 cues, gamma power & $82.16^{* * *}$ & 41.54 & 0.60 & $2.52+$ & 0.13 \\
Alpha power slope, old items & $13.27^{* * *}$ & 1.41 & 0.66 & $7.56^{* *}$ & 0.10 \\
Gamma power slope, old items & $7.15^{* *}$ & 10.77 & 0.12 & $5.08^{*}$ & \\
\hline
\end{tabular}


Table 4: Results of the regression analyses examining change in the behavioral and EEC variables as a function of change in the blood iron biomarkers (analyses for plausibility). Note that if a variable does not appear in this table, no acceptable regression model was identifie for that variable.

\begin{tabular}{|c|c|c|c|c|c|}
\hline \multirow[b]{2}{*}{ Task } & \multirow[b]{2}{*}{ Change variable } & \multicolumn{4}{|c|}{ Change in Blood Variable } \\
\hline & & Predictor & Intercept & $\hat{\beta}$ & $R^{2}$ \\
\hline \multicolumn{6}{|c|}{ Behavioral variables } \\
\hline GNG & Median RT & BdFe & 64 & 20 & 0.21 \\
\hline \multirow[t]{8}{*}{ ANT } & RT, 2 cues & $\begin{array}{l}\mathrm{Hb} \\
\mathrm{sFt}\end{array}$ & 55.8 & $\begin{array}{r}22.1 \\
3.9\end{array}$ & 0.17 \\
\hline & Alerting & $\mathrm{sFt}$ & 81.0 & 4.2 & 0.19 \\
\hline & RT, spatial cues & $\mathrm{sFt}$ & 8.7 & 4.0 & 0.18 \\
\hline & Orienting & $\mathrm{sFt}$ & -36.5 & 4.3 & 0.14 \\
\hline & $R T$, inconsistent flankers & $\mathrm{Hb}$ & -14.6 & 20.5 & 0.26 \\
\hline & & $\mathrm{sFt}$ & & 4.2 & \\
\hline & Conflict & $\mathrm{sFt}$ & -0.51 & 3.2 & 0.31 \\
\hline & & sTfR & & -83.1 & \\
\hline \multirow[t]{3}{*}{ CFE } & RT & $\mathrm{BdFe}$ & 6.86 & $17.12^{*}$ & 0.10 \\
\hline & Sensitivity & $\mathrm{BdFe}$ & 0.13 & 0.16 & 0.16 \\
\hline & Bias & $\mathrm{BdFe}$ & -0.03 & 0.07 & 0.11 \\
\hline \multirow[t]{3}{*}{ CRT } & RT, new items & $\mathrm{BdFe}$ & 47 & -32 & 0.11 \\
\hline & $\mathrm{RT}$, old items & $\mathrm{BdFe}$ & 44 & -23 & 0.15 \\
\hline & PCC & $\mathrm{sFt}$ & 30.00 & 0.78 & 0.11 \\
\hline \multicolumn{6}{|c|}{ EEG variables } \\
\hline \multirow{2}{*}{ SRT } & N1 amplitude & $\mathrm{Hb}$ & 0.86 & 0.95 & 0.11 \\
\hline & & BdFe & & 0.46 & \\
\hline \multirow[t]{2}{*}{ GNG } & P1 amplitude & $\mathrm{sFt}$ & -1.4 & 0.08 & 0.13 \\
\hline & $\mathrm{N}$ ! amplitude & $\mathrm{sFt}$ & -0.93 & 0.11 & 0.35 \\
\hline \multirow[t]{6}{*}{ ANT } & N1 amplitude, 2 cues & $\mathrm{sFt}$ & 1.61 & 0.05 & 0.10 \\
\hline & N1 amplitude, orienting & $\mathrm{sFt}$ & -1.23 & 0.09 & 0.21 \\
\hline & P3 amplitude, orienting & $\mathrm{sFt}$ & -1.03 & 0.03 & 0.10 \\
\hline & P3 amplitude, incongruent flankers & $\mathrm{sFt}$ & 1.78 & 0.06 & 0.25 \\
\hline & N1 amplitude, conflict & $\mathrm{sFt}$ & -0.49 & 0.05 & 0.23 \\
\hline & Gamma power, 2 cues & $\mathrm{sFt}$ & -1.02 & 0.05 & 0.15 \\
\hline & Interaction contrast, N1 amplitude & $\mathrm{sFt}$ & 1.01 & 0.05 & 0.15 \\
\hline \multirow[t]{3}{*}{ CRT } & P3 amplitude, old items 4 cues & $\mathrm{sFt}$ & 1.24 & 0.03 & 0.10 \\
\hline & Gamma power, old items, 4 cues & $\mathrm{sFt}$ & 5.49 & 0.11 & 0.10 \\
\hline & Gamma slope, old items 4 cues & $\mathrm{sFt}$ & 1.55 & 0.07 & 0.12 \\
\hline
\end{tabular}


Table 5: Parameters of the mediation models selected for the three composite variables. Notes: pred. $=$ predictor; int. $=$ intercept; $+=0.05 \leq p<0.10, *=p<0.05, * *=p<$ $0.01, * * *=p<0.001$.

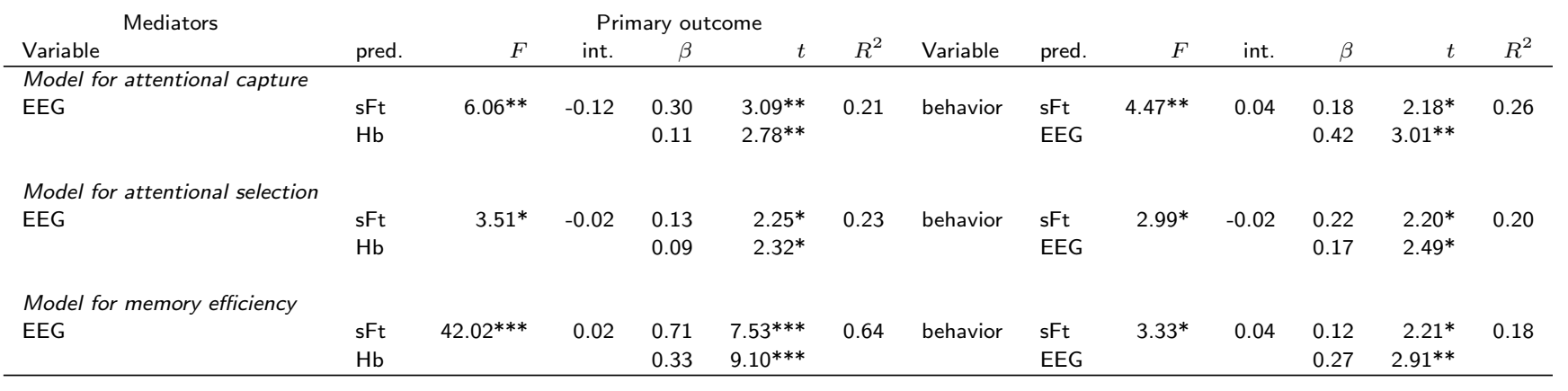


Additional Files

Additional file 1

Additional file 1 contains procedural details for each of the cognitive tasks as well as the details of the pre-processing of the EEG data. 


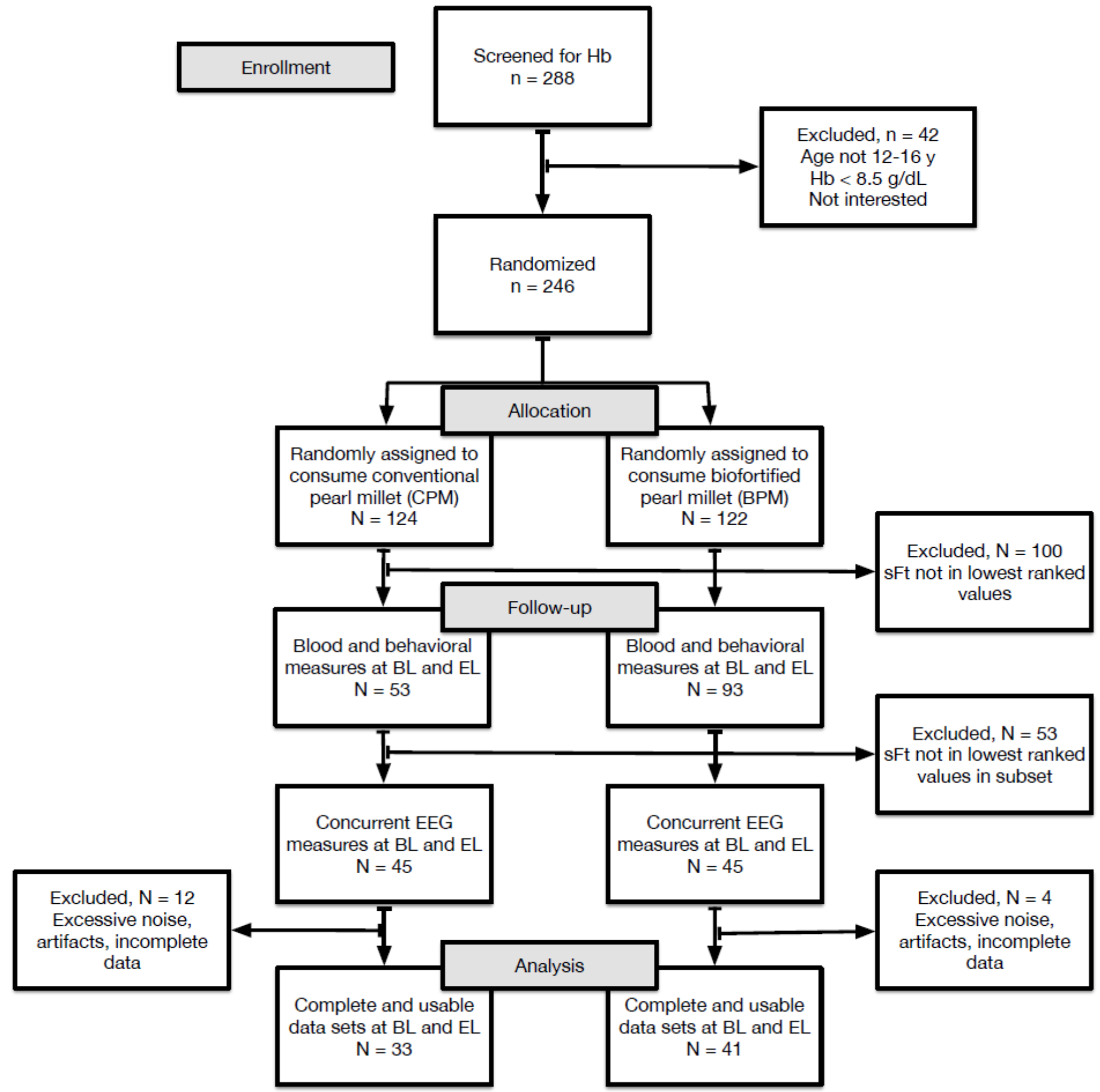

Figure 1

CONSORT diagram for the study. 


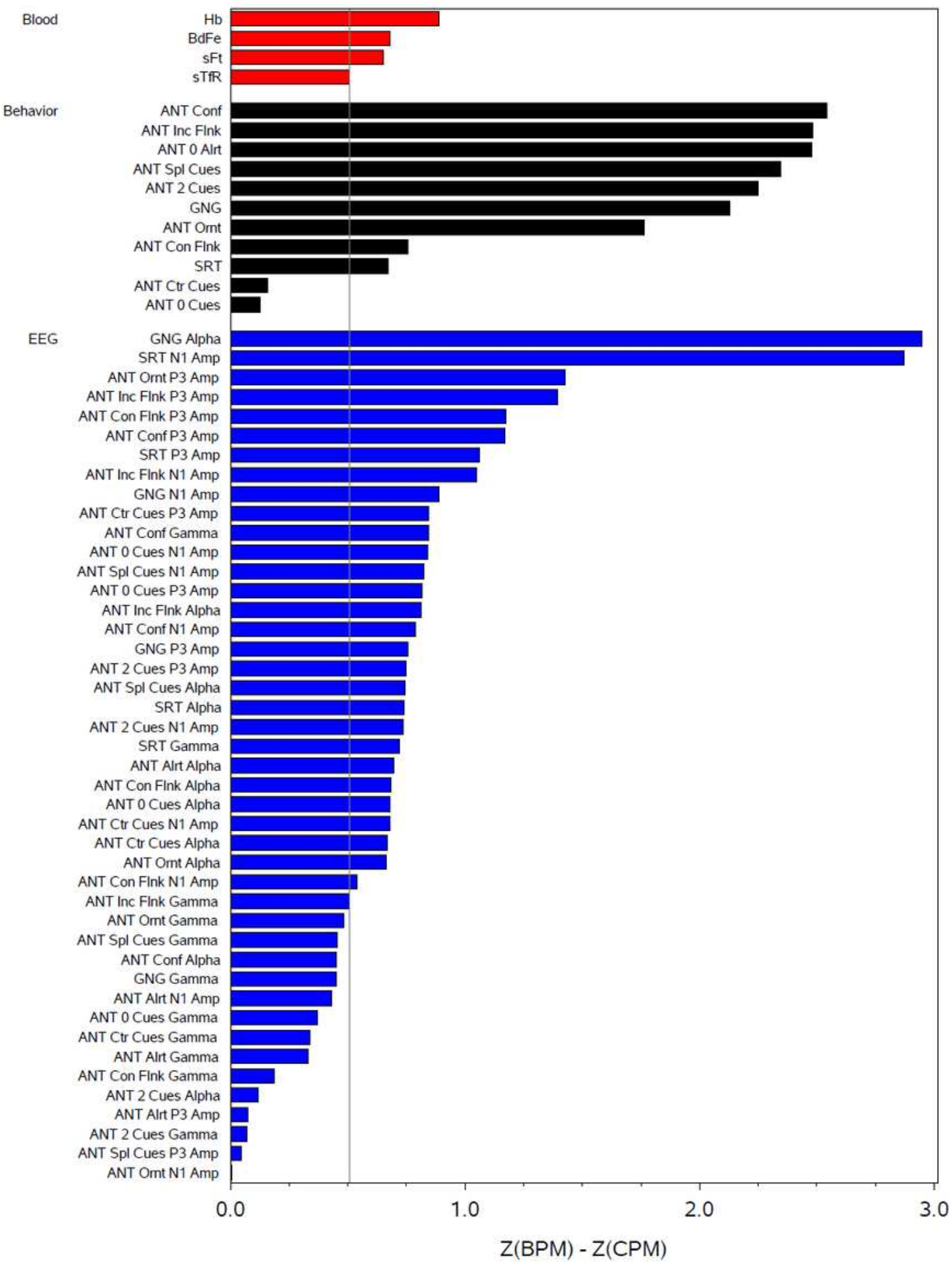

\section{Figure 2}

Magnitude of the difference in the normalized amount of change observed for those consuming the biofortified pearl millet (BPM) and those consuming the control pearl millet (CPM), on each of the blood, behavior, and EEG variables for the attention tasks. The reference lines denote the smallest amount of change observed for the blood biomarkers. 


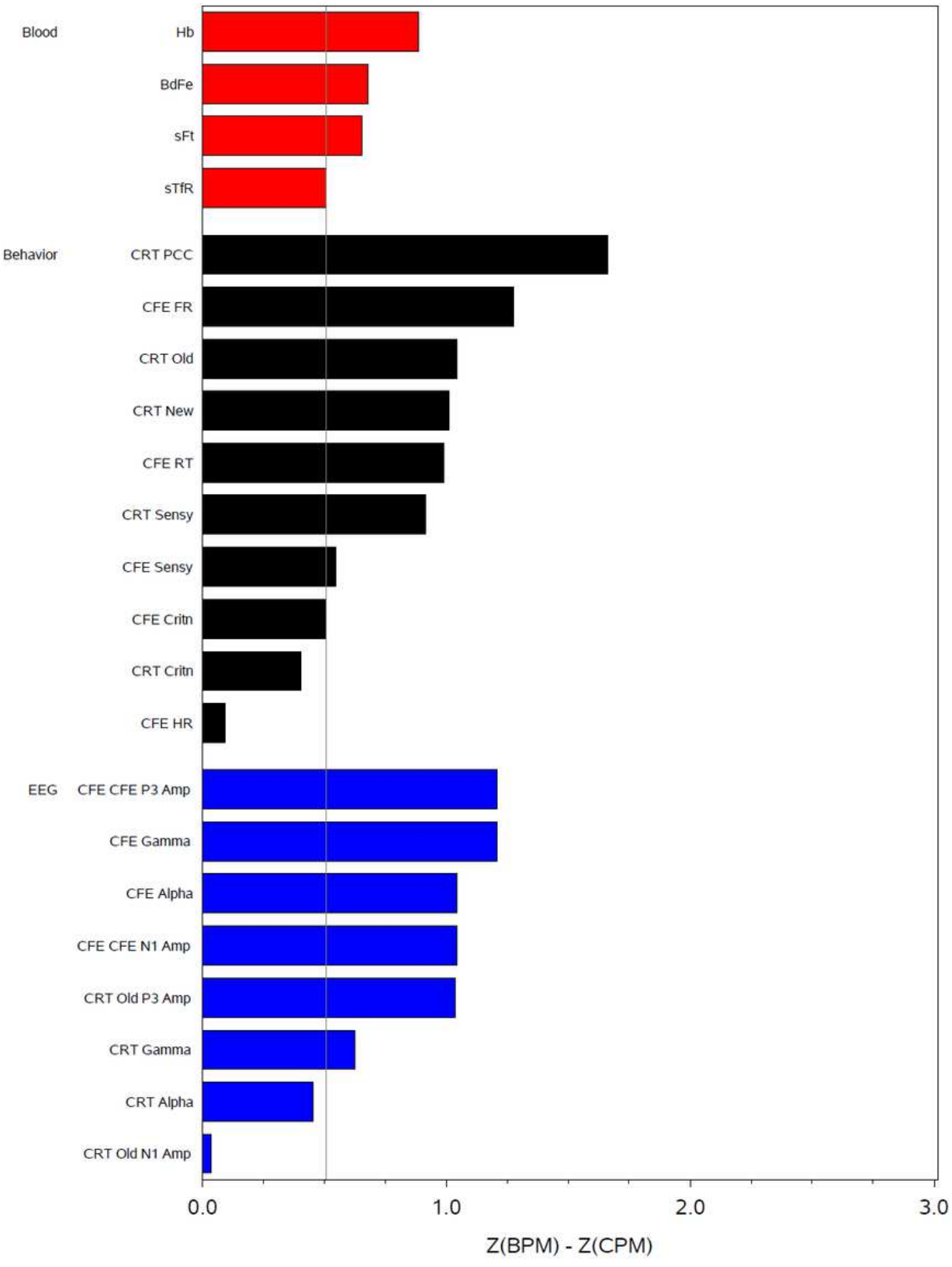

\section{Figure 3}

Magnitude of the difference in the normalized amount of change observed for those consuming the biofortified pearl millet (BPM) and those consuming the control pearl millet (CPM), on each of the blood, behavior, and EEG variables for the memory tasks. The reference lines denote the smallest amount of change observed for the blood biomarkers. 


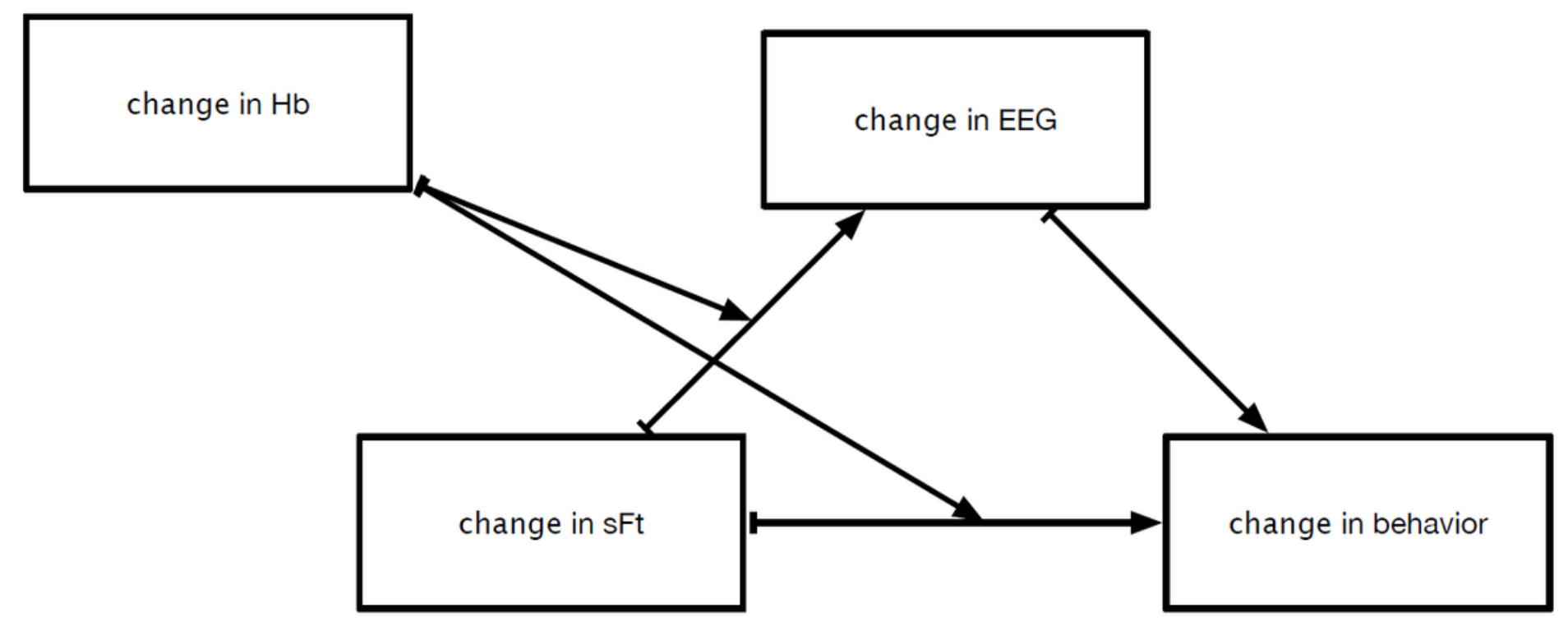

Figure 4

Common form for the best-fitting mediation models with effect modifiers. Change refers to change from baseline to endline. In all cases (see Table 5, all parameters were positive.

\section{Supplementary Files}

This is a list of supplementary files associated with this preprint. Click to download.

- additionalfile1.pdf 